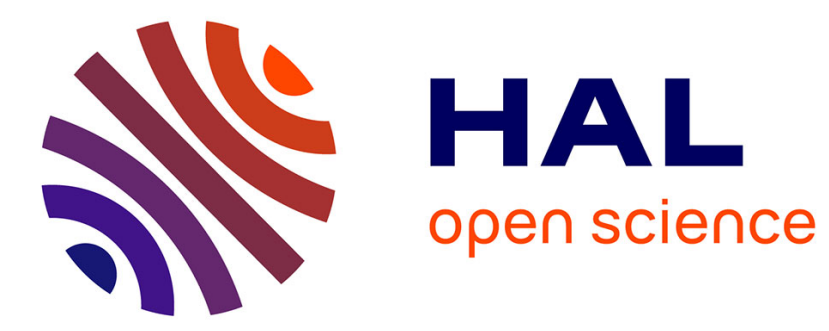

\title{
Drop Shaping by Laser-Pulse Impact
}

\author{
Alexander L. Klein, Wilco Bouwhuis, Claas Willem Visser, Henri Lhuissier, \\ Chao Sun, Jacco H. Snoeijer, Emmanuel Villermaux, Detlef Lohse, Hanneke \\ Gelderblom
}

\section{- To cite this version:}

Alexander L. Klein, Wilco Bouwhuis, Claas Willem Visser, Henri Lhuissier, Chao Sun, et al.. Drop Shaping by Laser-Pulse Impact. Physical Review Applied, 2015, 3 (4), 10.1103/PhysRevApplied.3.044018 . hal-01348634

\section{HAL Id: hal-01348634 https://hal.science/hal-01348634}

Submitted on 31 Jan 2017

HAL is a multi-disciplinary open access archive for the deposit and dissemination of scientific research documents, whether they are published or not. The documents may come from teaching and research institutions in France or abroad, or from public or private research centers.
L'archive ouverte pluridisciplinaire HAL, est destinée au dépôt et à la diffusion de documents scientifiques de niveau recherche, publiés ou non, émanant des établissements d'enseignement et de recherche français ou étrangers, des laboratoires publics ou privés. 


\title{
Drop Shaping by Laser-Pulse Impact
}

\author{
Alexander L. Klein, ${ }^{1, *}$ Wilco Bouwhuis, ${ }^{1}$ Claas Willem Visser, ${ }^{1}$ Henri Lhuissier, ${ }^{2}$ Chao Sun, ${ }^{1}$ Jacco H. Snoeijer, ${ }^{1,3}$ \\ Emmanuel Villermaux, ${ }^{4,5}$ Detlef Lohse, ${ }^{1, \dagger}$ and Hanneke Gelderblom ${ }^{1, ٪}$ \\ ${ }^{1}$ Physics of Fluids Group, Faculty of Science and Technology, MESA ${ }^{+}$Institute, University of Twente, \\ P.O. Box 217, 7500 AE Enschede, The Netherlands \\ ${ }^{2}$ Laboratoire Matière et Systèmes Complexes, Université Paris-Diderot/CNRS, \\ F-75205 Paris Cedex 13, France \\ ${ }^{3}$ Mesoscopic Transport Phenomena, Eindhoven University of Technology, Den Dolech 2, \\ 5612 AZ Eindhoven, The Netherlands \\ ${ }^{4}$ IRPHE, Aix-Marseille Université, 13384 Marseille Cedex 13, France \\ ${ }^{5}$ Institut Universitaire de France, 75005 Paris, France
}

(Received 23 January 2015; revised manuscript received 27 March 2015; published 28 April 2015)

\begin{abstract}
We show how the deposition of laser energy induces propulsion and strong deformation of an absorbing liquid body. Combining high speed with stroboscopic imaging, we observe that a millimeter-sized dyed water drop hit by a millijoule nanosecond laser pulse propels forward at several meters per second and deforms until it eventually fragments. The drop motion results from the recoil momentum imparted at the drop surface by water vaporization. We measure the propulsion speed and the time-deformation law of the drop, complemented by boundary-integral simulations. The drop propulsion and shaping are explained in terms of the laser-pulse energy, the drop size, and the liquid properties. These findings are, for instance, crucial for the generation of extreme ultraviolet light in nanolithography machines.
\end{abstract}

DOI: 10.1103/PhysRevApplied.3.044018

\section{INTRODUCTION}

A laser-induced phase change in liquids can lead to a violent response: deformation and disruption of the liquid body followed by the ejection of matter. The complete vaporization or even explosion of micrometer-sized drops can result from the linear absorption of laser energy [1-3]. Self-focusing and dielectric breakdown may lead to plasma formation in transparent drops [4-7]. Laser impact has been used to generate liquid motion by vaporization or plasma formation in confined geometries [8-10], sessile drops [11], and biological matter [12-14].

Here, we show how the absorption of laser energy by an unconfined liquid drop induces a rapid phase change (see Fig. 1), which in turn controls the propulsion, expansion, and fragmentation of the drop. A key application of the drop shaping by laser impact is found in laser-produced plasma light sources for extreme ultraviolet (EUV) nanolithography $[15,16]$. In these sources the shape, position, and stability of a liquid-tin body directly affect the conversion efficiency of liquid tin to a plasma that emits EUV light.

The detailed understanding of the hydrodynamic response of an opaque liquid drop to laser impact poses two fundamental challenges. First, one needs to resolve how momentum is transferred from the laser to the drop. Second, the subsequent deformation dynamics

\footnotetext{
*alexludwig.klein@utwente.nl

†.lohse@utwente.nl

th.gelderblom@utwente.nl
}

and fragmentation of the drop after impact have to be quantified. Although drop impact onto a solid substrate has been studied thoroughly (for a selection, see, e.g., Refs. [18-24]), no consensus on the deformation dynamics has yet been reached and only few studies [19,20, 25-27] focused on the fragmentation.

\section{EXPERIMENTAL METHODS}

Our model system consists of a highly absorbing drop that is hit by a pulsed laser beam. In Fig. 2 an overview of the experimental setup is shown. The drop detaches from a capillary, falls, and relaxes to a spherical shape with radius
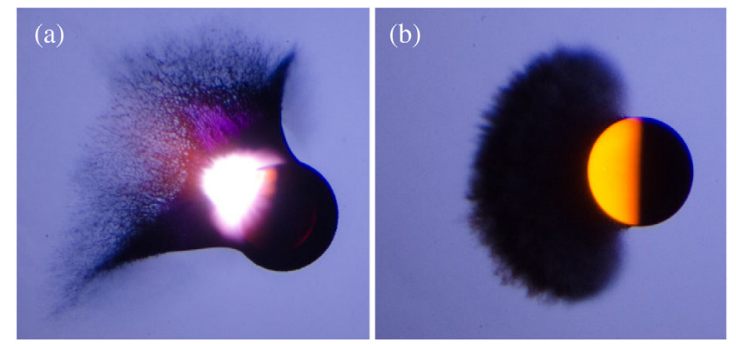

FIG. 1. Laser pulses $(\lambda=532 \mathrm{~nm})$ impacting from the left on magenta-dyed water drops of radius $R_{0}=0.9 \mathrm{~mm}$. Images are taken $30 \mu \mathrm{s}$ after impact with a color camera and diffusive backlight illumination. (a) White plasma glow [17] and violent ablation from the drop induced by a focused laser beam. (b) Fluorescence of the dye and ablation at the drop surface due to local boiling induced by a uniform laser irradiation. 


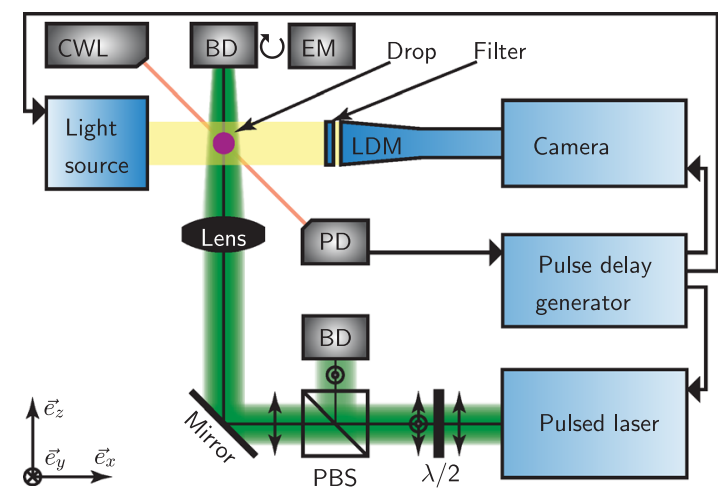

FIG. 2. Sketch of the experimental setup in top view. The drop [black (magenta) ink, IJC-5900 (5920) by Sensient] is generated with a capillary tube connected to a syringe pump (PHD2000 by Harvard Apparatus, not shown). A continuous-wave laser (CWL, CPS196 by Thorlabs) and a photodiode (PD, PDA36A by Thorlabs) serve as a light barrier to trigger on the falling drop. The lab equipment is synchronized by a high-precision pulsedelay generator (BNC575 by Berkeley Nucleonics) according to the indicated signal path. The pulsed laser is a frequency-doubled $\mathrm{Nd}$ : YAG laser (Evergreen 140 by Quantel) with a pulse duration $\tau_{p}=10 \mathrm{~ns}$ emitting at a wavelength $\lambda=532 \mathrm{~nm}$. Attenuation of the laser energy is accomplished by a zero-order half-wave plate $(\lambda / 2)$, a polarizing beam splitter (PBS), and a beam dump (BD). The laser-pulse energy is measured by an energy meter (EM, QE12 by gentec-eo). The circle and arrow symbols along the optical axis respectively indicate the $S$ and $P$ component of the linearly polarized laser beam that is focused by a plano-convex lens with a focal length of $f=125 \mathrm{~mm}$. Side-view images ( $y z$ plane) are taken with a long-distance microscope (LDM, 12× zoom by Navitar), a high speed camera (FASTCAM SA-X2 by Photron), and a continuous light source (LS-M352A by Sumita). Stroboscopic images are acquired by a CCD camera (PCO1300 by PCO AG) combined with a light source (NANOLITE KL-K by HSPS) that delivers a high-intensity light pulse of $8 \mathrm{~ns}$. A notch filter protects the imaging equipment from scattered laser light.

$R_{0}=0.9 \mathrm{~mm}$. While it falls down, the drop masks a photodiode that then generates a reference trigger for the pulsed laser, cameras, and light source. The $\vec{e}_{z}$ axis of the laser beam is aligned orthogonally to the $\vec{e}_{y}$ axis defined by the falling drop and the $\vec{e}_{x}$ axis of the imaging optics. The $x z$ plane in which the laser beam propagates is below the plane of the trigger laser and the pinch-off point at the capillary tube.

The drop consists of dyed water with a density $\rho=$ $998 \mathrm{~kg} / \mathrm{m}^{3}$ and surface tension $\gamma=72 \mathrm{mN} / \mathrm{m}$ assumed to be equal to the properties of pure water. The typical penetration depth of the laser light into the dyed drop is $\delta \sim 10 \mu \mathrm{m} \ll R_{0}[10]$, which ensures that the laser energy is absorbed in a thin layer close to the drop surface. The laser-pulse energy is varied between 0 and $120 \mathrm{~mJ}$ by an optical attenuator based on a half-wave plate and a polarizing beam splitter. The relation between the laser-pulse energy at the drop location and the settings of the attenuator is determined in separate measurements, for which the top beam dump shown in Fig. 2 is replaced by an energy meter. A focusing lens decreases the beam diameter to twice the drop size in order to achieve a uniform but high-intensity illumination of the drop. To ensure the drop is placed at the center of the laser beam, the drop position is optimized such that the drop-shape evolution is axisymmetric with respect to $\vec{e}_{z}$ and the propulsion speed is maximum.

The energy $E$ that is actually absorbed by the drop is computed from a beam-profile measurement and ray tracing [28]. The typical beam fluence $1 \mathrm{~J} / \mathrm{cm}^{2}$ is well below the dielectric breakdown and self-focusing thresholds reported for water with focused nanosecond laser pulses $[8,17]$. Consistently, we observe a plasma only when the laser beam is tightly focused inside the drop [Fig. 1(a), see also Refs. $[12,13]]$, but not for a uniform irradiation [Fig. 1(b)]. To visualize the wavelengths in the visible spectrum that are emitted by the drop shown in Fig. 1, we use a magenta-colored ink as a dye and a color camera. For all experiments leading to quantitative results we use a black-colored ink to suppress fluorescence.

The postimpact dynamics of the drop [Fig. 3(a)] is observed from a side view ( $\vec{e}_{z}$ axis in Fig. 2$)$ with a longdistance microscope, a high-speed camera operated at a frame rate of 20000 frames/s, and a continuous light source. Detailed information in the first microseconds after impact is obtained by operating a camera in stroboscopic mode with a flash lamp that delivers a high-intensity light pulse of $8 \mathrm{~ns}$ [Fig. 3(b)]. We record stroboscopic videos by performing a single-impact experiment per video frame while changing the time delay between the laser impact and the pulsed light source. For both cameras used, the size of the field of view is $16 \times 10 \mathrm{~mm}^{2}$, which yields a pixel resolution of $16 \mu \mathrm{m}$ per pixel.

\section{RESULTS AND INTERPRETATION}

The drop dynamics for different pulse energies is shown in Fig. 3. On impact, the surface of the drop hit by the laser emits a shock wave into the air [Fig. 3(b)]. The shock wave is followed by the ejection of a mist cloud of small drops that is visible as a gray-to-black haze in the images and persists for several microseconds. Subsequently, the mist is expelled while the drop propels in the opposite direction [Fig. 3(a)]. At the same time the drop flattens and expands in the radial direction before it either retracts, for low pulse energy, or fragments, for large energy.

We quantify the drop motion by measuring the displacement $Z(t)$ of the drop center of mass and the drop radius $R(t)$ [defined in Fig. 3(a)] for the first milliseconds after impact. As Fig. 4(a) shows, the drop is propelled at a constant speed $U$ that increases with increasing pulse energy up to $2.0 \mathrm{~m} / \mathrm{s}$. The accompanying deformation of the drop occurs on the inertial time scale $\tau_{i}=R_{0} / U \sim 10^{-4}$ to $10^{-3} \mathrm{~s}$ [Fig. 4(b)] and is eventually slowed down by 


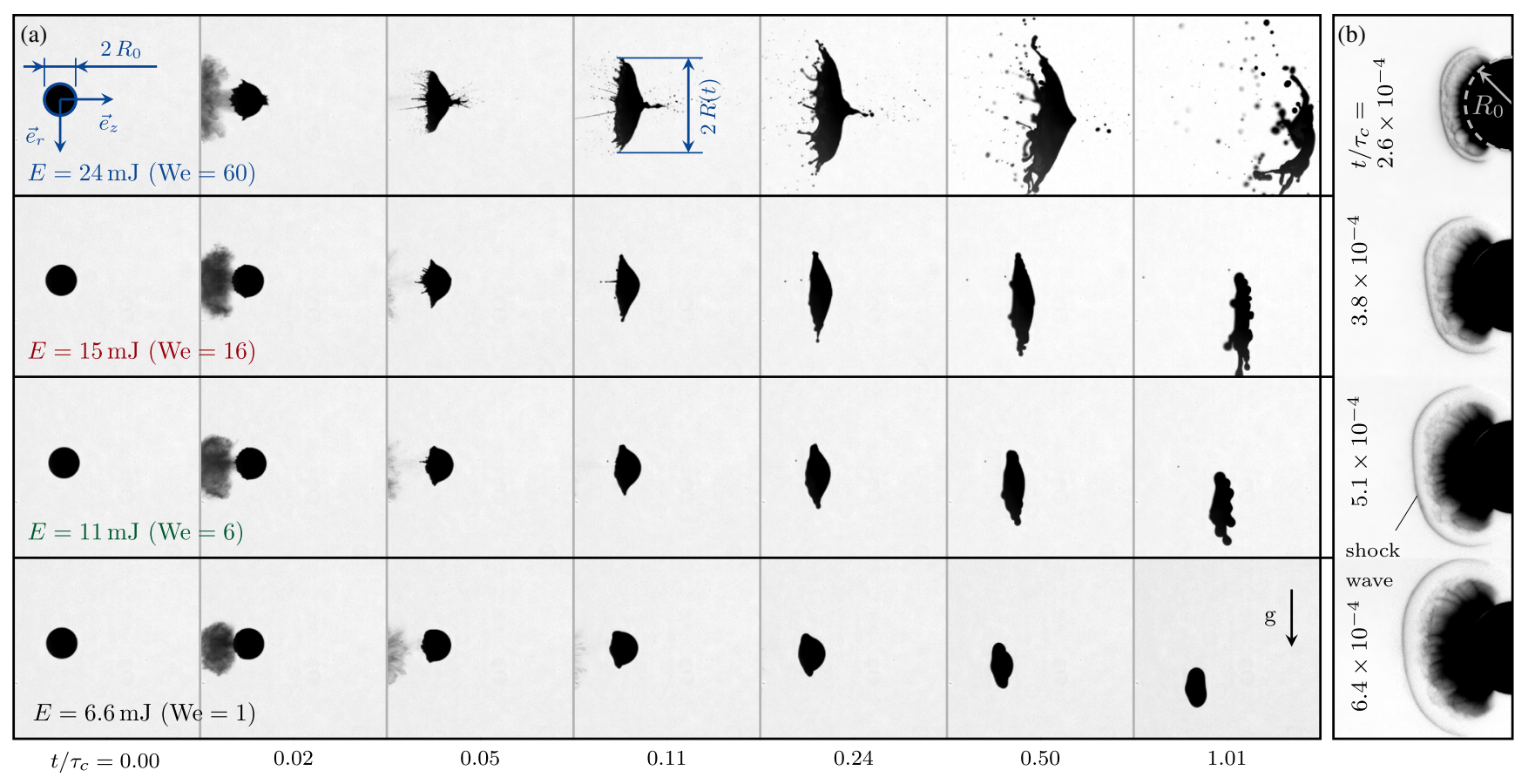

FIG. 3. Side view of a dyed water drop with an initial radius $R_{0}=0.9 \mathrm{~mm}$ hit at $t=0$ by a laser pulse propagating from left to right $\left(\vec{e}_{z}\right)$. (a) Drop-shape dynamics for pulse energies increasing from bottom to top. $E$ is the energy that is actually absorbed by the drop, We is the Weber number of the propelled drop (see text). The images are taken at a frame rate of $20000 \mathrm{frames} / \mathrm{s}\left(\tau_{c}=3.5 \mathrm{~ms}\right)$. As the laser ablates the front of the drop a mist cloud is ejected backward $\left(-\vec{e}_{z}\right)$ while the remainder of the drop is propelled forward $\left(\vec{e}_{z}\right)$ and expands radially $\left(\vec{e}_{r}\right)$. For small $E$ the drop retracts after the initial expansion and no breakup occurs. For $E=24 \mathrm{~mJ}$ the edge destabilizes before it retracts and the drop fragments. (b) Close-up view of the drop surface for $E=24 \mathrm{~mJ}$ revealing the shock wave in the air and the mist cloud development at early times (increasing from top to bottom).

surface tension on the capillary time scale $\tau_{c}=\sqrt{\rho R_{0}^{3} / \gamma}=$ $3.5 \mathrm{~ms}$. Both the initial deformation rate $\tau_{i}^{-1}$ and the maximal extension $R_{\max }$ increase with increasing pulse energy. We emphasize the clear separation of time scales

$$
\tau_{p} \ll \tau_{e} \ll \tau_{i}<\tau_{c}
$$

between the successive steps, namely, the laser pulse, the ejection of matter (on the time scale $\tau_{e} \sim 10^{-5} \mathrm{~s}$ ), the initial deformation of the drop, and its capillary retraction.

To explain the relation between the drop-propulsion speed, the radial expansion, and the laser energy one needs to understand the mechanism that propels the drop. Surely, both the optical-radiation pressure from the laser and the thermal-radiation pressure caused by the heating of the drop surface are insignificant $[29,30]$. The motion actually results from the recoil due to the partial vaporization of the drop: since the highly absorbent dye ensures that the laser energy is absorbed in a superficial layer on one side of the drop, the vapor expulsion is mainly unidirectional and consequently transfers momentum to the remainder of the drop.

The light energy is absorbed by a liquid mass $\sim \rho R_{0}^{2} \delta$ set by the penetration depth of the laser. On the time scale $\tau_{e}$, both diffusive and radiative heat transfers are negligible (the thermal diffusion length is much smaller than $\delta$ [31]).
Since the beam profile is flat, and neither the focusing due to the drop-interface curvature nor nonlinear optical effects (self-focusing or electric breakdown) are significant, we consider the energy deposition in the superficial layer to be close to uniform. This energy is sufficient to heat the liquid from the ambient temperature $T_{0}=293 \mathrm{~K}$ to the boiling temperature $T_{b} \simeq 393 \mathrm{~K}$, but not to vaporize all of it. Only a certain fraction $\beta$ actually vaporizes. The energy balance therefore reads $E \sim \rho R_{0}^{2} \delta\left[c_{v}\left(T_{b}-T_{0}\right)+\beta \Delta H\right]$, where $c_{v}=4.0 \mathrm{~kJ} /(\mathrm{kg} \mathrm{K})$ and $\Delta H=2.25 \mathrm{MJ} / \mathrm{kg}$ are, respectively, the specific heat capacity and latent heat of vaporization of the liquid.

In all of our experiments a mist cloud is observed, which is a clear signal of a local boiling of the drop. We therefore assume that to get propulsion, a threshold energy $E_{\mathrm{th}} \sim$ $\rho R_{0}^{2} \delta c_{v}\left(T_{b}-T_{0}\right) \approx 3 \mathrm{~mJ}$ has to be absorbed by the superficial layer to heat the liquid to the boiling point, which is in good agreement with the threshold for propulsion observed in our experiments [Fig. 5(a)]. Any additional energy deposited in the superficial layer is used to vaporize a mass of liquid $m \sim \beta \rho R_{0}^{2} \delta \sim\left(E-E_{\text {th }}\right) / \Delta H$. An upper limit for the proposed scaling is given by $E / E_{\mathrm{th}} \sim 1+\Delta H /$ $\left[c_{v}\left(T_{b}-T_{0}\right)\right] \approx 8$, in which case the absorbed energy is sufficient to evaporate the entire heated liquid layer (i.e., $\beta=1$ ). Any increase in $E$ beyond this point would 


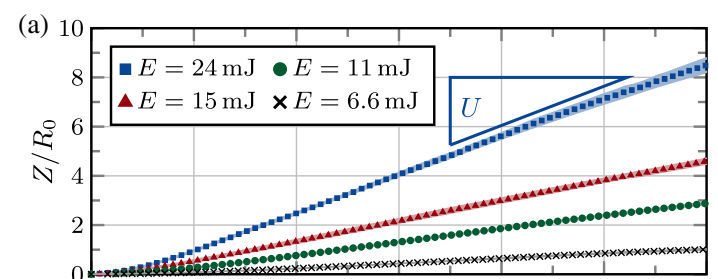

(b)

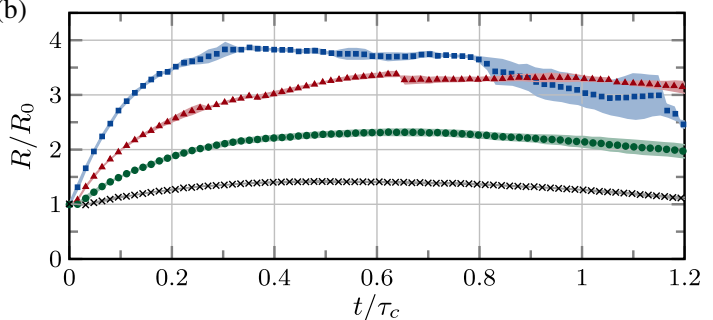

FIG. 4. Center-of-mass displacement $Z$ (along $\vec{e}_{z}$, see Fig. 3) (a) and radial expansion $R$ (b) as a function of time for different absorbed energies. The corresponding image sequences are shown in Fig. 3. Each point is averaged over two experiments and the shaded area indicates the difference between the two. The apparent acceleration in $Z$ for $t / \tau_{c}<0.2$ is an artifact of the method used to determine the center-of-mass position [28]. The large deviation in $R$ for $E=24 \mathrm{~mJ}$ illustrates the statistical nature of the fragmentation. For $E=15 \mathrm{~mJ}$, events of drop ejection from the edge are visible at $t / \tau_{c}=0.24,0.36$, and 0.63 .

lead to a superheated or even a critical phase, in which case the opaque mist cloud would not be observed [13].

For $0<\beta<1$, which is the case of our experiments, the remaining part of the heated layer that is not vaporized is expelled as a mist of small drops. We assume that the liquid vaporizes at $T_{b}$ and that the vapor is expelled at the thermal speed $u=\sqrt{k_{B} T_{b} / \mu} \approx 400 \mathrm{~m} / \mathrm{s}$, where $k_{B} \simeq$ $1.38 \times 10^{-23} \mathrm{~J} / \mathrm{K}$ is the Boltzmann constant, and $\mu=$ $2.99 \times 10^{-26} \mathrm{~kg}$ is the molecular mass of water. This expelled vapor propels the remainder of the drop. Momentum conservation $m u=\rho R_{0}^{3} U$ yields

$$
U \sim \frac{E-E_{\text {th }}}{\rho R_{0}^{3} \Delta H} u
$$

that is, an increase in $U$ proportional to that in $E$. Figure 5(a) shows that this scaling argument, with a prefactor of 0.4 , is in good agreement with our experimental data.

With a description of the propulsion at hand, we now turn to the drop deformation. The expansion dynamics is directly affected by surface tension, which promotes the retraction and possibly the fragmentation of the drop. The key parameter describing the expansion is therefore the Weber number of the motion induced by the laser $\mathrm{We}=\rho R_{0} U^{2} / \gamma$, which compares the drop-displacement kinetic energy to its surface energy. In our experiments $1 \leq \mathrm{We} \leq 60$. The impulsive acceleration of our drop from 0 to $U$ is similar to the impulsive stop of a drop impacting a solid with velocity $U$. We therefore use the momentum-based scaling derived
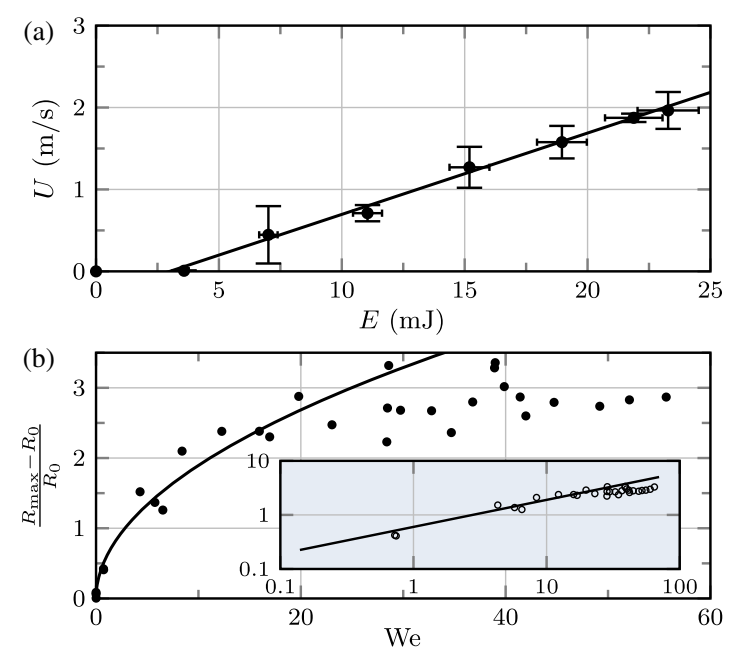

FIG. 5. (a) Propulsion speed of the drop as a function of the absorbed laser energy. Each point represents at least four experiments; the error bars indicate the standard deviation. The solid line is Eq. (2) with a prefactor of 0.4. (b) Maximal relative expansion $R_{\max } / R_{0}-1$ for individual experiments as a function of the Weber number in linear and logarithmic (inset) scales. The solid line is Eq. (3) with a prefactor of 0.6. For large Weber numbers a saturation is observed due to the fragmentation of the sheet.

by $[18,20]$ for drop impact on solids to express the maximal radial expansion

$$
\frac{R_{\max }-R_{0}}{R_{0}} \sim \mathrm{We}^{1 / 2} \sim \sqrt{\frac{\rho R_{0} u^{2}}{\gamma}} \frac{E-E_{\mathrm{th}}}{\rho R_{0}^{3} \Delta H},
$$

in which the expression in terms of $E$ directly comes from Eq. (2). Expression (3), with a prefactor of 0.6, is in good agreement with our experimental data up to $\mathrm{We} \sim 40$, when the drop starts fragmenting and the maximum expansion saturates [see Fig. 5(b)]. The scaling (3) has already been observed for drop impact onto solid substrates with negligible friction [20]. The present setup is, however, fundamentally different since, as mentioned above, the typical impact time scale, during which the drop accelerates, is decoupled from the inertial time scale: $\tau_{e} \ll \tau_{i}$.

\section{NUMERICAL RESULTS}

To confirm that the interaction of the laser pulse with the drop can be modeled as a short recoil-pressure pulse exerted on the drop surface, we perform boundary-integral (BI) simulations [32-34]. We assume that the flow inside the drop is inviscid, irrotational, and incompressible, and solve the resulting Laplace equation for the flow potential. The method assumes axisymmetry and therefore cannot be used to study the eventual fragmentation of the drop, but it does capture the initial phase of the drop deformation.

The laser pulse is modeled by applying a pressure boundary condition at the drop surface for a time duration 


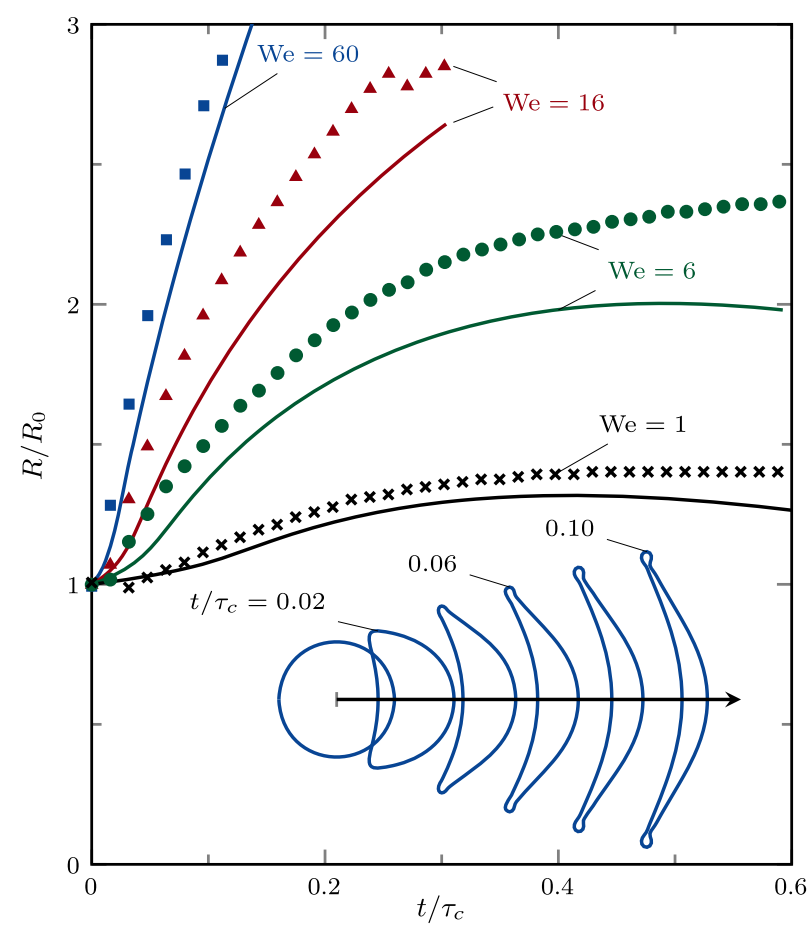

FIG. 6. Radial expansion of the drop: experiments (markers) and BI simulations (solid lines). The corresponding image sequences are shown in Fig. 3. The inset shows the drop-shape evolution from $\mathrm{BI}$ for $\mathrm{We}=60$ with an exaggerated centerof-mass displacement to separate the successive shapes. The simulations are stopped when the local sheet thickness becomes too thin to resolve the dynamics accurately.

$\tau_{e} \ll \tau_{i}$. We use a Gaussian pressure profile with a length scale based on the measured laser-beam profile and a pressure scale set to match the propulsion velocity observed in the experiment. This pressure scale is prescribed by the momentum conservation $p R_{0}^{2} \tau_{e} \sim \rho R_{0}^{3} U$ (the prefactor can be obtained analytically [35]). From Eq. (2) this recoil pressure can readily be expressed in terms of the absorbed energy.

The numerical drop-shape evolution is shown in Fig. 6. It illustrates the added value of the simulations: not only the two-dimensional projection of the drop shape, but also the spatial and temporal evolution of the sheet thickness can be extracted, which is crucial when it comes to the study of fragmentation [20]. Moreover, Fig. 6 shows that the BI model quantitatively predicts the radial drop expansion observed for different Weber numbers. This confirms that a pressure pulse applied at the drop surface for a time much shorter than the hydrodynamic time scales $\left(\tau_{i}\right.$ and $\left.\tau_{c}\right)$ is indeed sufficient to describe the hydrodynamic response of a drop to the impact of a laser pulse.

\section{CONCLUSIONS}

We show that an opaque free-falling drop hit by a laser pulse propels and expands until fragmentation occurs.
In the present case the laser energy is absorbed in a superficial layer of the drop such that the deposited energy per unit mass $E / \rho R_{0}^{2} \delta \sim 0.1$ to $1 \mathrm{MJ} / \mathrm{kg}$ is comparable to the specific latent heat of vaporization. As a consequence, drop motion is induced by the recoil due to vaporization on the face of the drop that is hit by the laser. This results in a propulsion speed and a maximal radius of expansion that are both proportional to the pulse energy. The expansion dynamics is limited by surface tension and is similar to that of a drop impacting a solid, although with a laser-pulse momentum transfer takes place on a much shorter time scale. Laser-induced drop fragmentation and the influence of the beam focusing require detailed studies and are left for future work [35]. All results reported here should transpose directly to the shaping of liquid-tin drops in EUV light sources. In a regime where a plasma is generated the propulsion mechanism may change, however, the Weber number remains the key parameter governing the hydrodynamic response.

\section{ACKNOWLEDGMENTS}

We thank Chris Lee, Andrea Prosperetti, and Guillaume Lajoinie for fruitful discussions. This work is part of an Industrial Partnership Programme of the Foundation for Fundamental Research on Matter (FOM), which is financially supported by the Netherlands Organization for Scientific Research (NWO). This research programme is cofinanced by ASML. W. B. and J.H.S. acknowledge support from NWO through VIDI Grant No. 11304.

[1] R. G. Pinnick, A. Biswas, R. L. Armstrong, S. G. Jennings, J. D. Pendleton, and G. Fernández, Micron-sized droplets irradiated with a pulsed $\mathrm{CO}_{2}$ laser: Measurement of explosion and breakdown thresholds, Appl. Opt. 29, 918 (1990).

[2] P. Kafalas and A. P. Ferdinand, Fog droplet vaporization and fragmentation by a 10.6- $\mu$ m laser pulse, Appl. Opt. 12, 29 (1973).

[3] P. Kafalas and J. Herrmann, Dynamics and energetics of the explosive vaporization of fog droplets by a $10.6-\mu \mathrm{m}$ laser pulse, Appl. Opt. 12, 772 (1973).

[4] J.-Z. Zhang, J. K. Lam, C. F. Wood, B.-T. Chu, and R. K. Chang, Explosive vaporization of a large transparent droplet irradiated by a high intensity laser, Appl. Opt. 26, 4731 (1987).

[5] C. Favre, V. Boutou, S. C. Hill, W. Zimmer, M. Krenz, H. Lambrecht, J. Yu, R. K. Chang, L. Woeste, and J.-P. Wolf, White-Light Nanosource with Directional Emission, Phys. Rev. Lett. 89, 035002 (2002).

[6] Y. Geints, A. Kabanov, G. Matvienko, V. K. Oshlakov, A. A. Zemlyanov, S.S. Golik, and O. A. Bukin, Broadband emission spectrum dynamics of large water droplets exposed to intense ultrashort laser radiation, Opt. Lett. 35, 2717 (2010). 
[7] A. Lindinger, J. Hagen, L. D. Socaciu, T. M. Bernhardt, L. Wóste, D. Duft, and T. Leisner, Time-resolved explosion dynamics of $\mathrm{H}_{2} \mathrm{O}$ droplets induced by femtosecond laser pulses, Appl. Opt. 43, 5263 (2004).

[8] A. Vogel, S. Busch, and U. Parlitz, Shock wave emission and cavitation bubble generation by picosecond and nanosecond optical breakdown in water, J. Acoust. Soc. Am. 100, 148 (1996).

[9] C. Sun, E. Can, R. Dijkink, D. Lohse, and A. Prosperetti, Growth and collapse of a vapour bubble in a microtube: The role of thermal effects, J. Fluid Mech. 632, 5 (2009).

[10] Y. Tagawa, N. Oudalov, C. W. Visser, I. R. Peters, D. van der Meer, C. Sun, A. Prosperetti, and D. Lohse, Highly Focused Supersonic Microjets, Phys. Rev. X 2, 031002 (2012).

[11] S. Thoroddsen, K. Takehara, T. G. Etoh, and C.-D. Ohl, Spray and microjets produced by focusing a laser pulse into a hemispherical drop, Phys. Fluids 21, 112101 (2009).

[12] A. Vogel and V. Venugopalan, Mechanisms of pulsed laser ablation of biological tissues, Chem. Rev. 103, 577 (2003).

[13] I. Apitz and A. Vogel, Material ejection in nanosecond Er: YAG laser ablation of water, liver, and skin, Appl. Phys. A 81, 329 (2005).

[14] V. Horneffer, N. Linz, and A. Vogel, Principles of laserinduced separation and transport of living cells, J. Biomed. Opt. 12, 054016 (2007).

[15] H. Mizoguchi, T. Abe, Y. Watanabe, T. Ishihara, T. Ohta, T. Hori, T. Yanagida, H. Nagano, T. Yabu, S. Nagai, G. Soumagne, A. Kurosu, K. M. Nowak, T. Suganuma, M. Moriya, K. Kakizaki, A. Sumitani, H. Kameda, H. Nakarai, and J. Fujimoto, 1st generation Laser-Produced Plasma source system for HVM EUV lithography, Proc. SPIE Int. Soc. Opt. Eng. 7636, 763608 (2010).

[16] V. Y. Banine, K. N. Koshelev, and G. H. P. M. Swinkels, Physical processes in EUV sources for microlithography, J. Phys. D 44, 253001 (2011).

[17] P. Kennedy, D. Hammer, and B. Rockwell, Laser-induced breakdown in aqueous media, Prog. Quantum Electron. 21, 155 (1997).

[18] C. Clanet, C. Béguin, D. Richard, and D. Quéré, Maximal deformation of an impacting drop, J. Fluid Mech. 517, 199 (2004).

[19] A. Yarin, Drop impact dynamics: Splashing, spreading, receding, bouncing..., Annu. Rev. Fluid Mech. 38, 159 (2006).

[20] E. Villermaux and B. Bossa, Drop fragmentation on impact, J. Fluid Mech. 668, 412 (2011).

[21] P. Tsai, M. Hendrix, R. Dijkstra, L. Shui, and D. Lohse, Microscopic structure influencing macroscopic splash at high Weber number, Soft Matter 7, 11325 (2011).

[22] C. W. Visser, P. E. Frommhold, S. Wildeman, R. Mettin, D. Lohse, and S. Chao, Dynamics of high-speed micro-drop impact: Numerical simulations and experiments at frame-toframe times below 100 ns, Soft Matter 11, 1708 (2015).

[23] T. Tran, H. J. J. Staat, A. Susarrey-Arce, T. C. Foertsch, A. Van Houselt, H. J. G. E. Gardeniers, A. Prosperetti, D. Lohse, and C. Sun, Droplet impact on superheated micro-structured surfaces, Soft Matter 9, 3272 (2013).

[24] G. Riboux and J. M. Gordillo, Experiments of Drops Impacting a Smooth Solid Surface: A Model of the Critical Impact Speed for Drop Splashing, Phys. Rev. Lett. 113, 024507 (2014).

[25] L. Xu, L. Barcos, and S. R. Nagel, Splashing of liquids: Interplay of surface roughness with surrounding gas, Phys. Rev. E 76, 066311 (2007).

[26] E. Villermaux, Fragmentation, Annu. Rev. Fluid Mech. 39, 419 (2007).

[27] E. Villermaux and B. Bossa, Single-drop fragmentation determines size distribution of raindrops, Nat. Phys. 5, 697 (2009).

[28] See Supplemental Material at http://link.aps.org/ supplemental/10.1103/PhysRevApplied.3.044018 for additional movies and technical details.

[29] The typical impulse $I$ exerted on the drop by the $3 \%$ reflected light from the surface scales as $I \sim E_{r} / c$, with $E_{r}$ the energy of the reflected light and $c$ the speed of light. This impulse would yield a typical drop speed $U \sim 10^{-7} \mathrm{~m} / \mathrm{s}$. The impulse due to thermal radiation from the hot drop surface scales as $I \sim \epsilon \sigma T^{4} R_{0}^{2} \tau_{p} / c$ with $\epsilon$ the emissivity and $\sigma$ the Stefan-Boltzmann constant. This impulse would yield $U \sim 10^{-14} \mathrm{~m} / \mathrm{s}$.

[30] J.-P. Delville, M. de Saint Vincent, R. Schroll, H. Chraïbi, B. Issenmann, R. Wunenburger, D. Lasseux, W. Zhang, and E. Brasselet, Laser microfluidics: Fluid actuation by light, J. Opt. A 11, 034015 (2009).

[31] On the time scale of vapor ejection $\tau_{e}$ heat typically diffuses over a length $\sqrt{\kappa \tau_{e}} \sim 10^{-6} \mathrm{~m} \ll \delta$, where $\kappa$ is the thermal diffusivity of water.

[32] R. Bergmann, D. van der Meer, S. Gekle, A. van der Bos, and D. Lohse, Controlled impact of a disk on a water surface: Cavity dynamics, J. Fluid Mech. 633, 381 (2009).

[33] S. Gekle, I. R. Peters, J. M. Gordillo, D. van der Meer, and D. Lohse, Supersonic Air Flow due to Solid-Liquid Impact, Phys. Rev. Lett. 104, 024501 (2010).

[34] W. Bouwhuis, R. C. A. van der Veen, T. Tran, D. L. Keij, K. G. Winkels, I. R. Peters, D. van der Meer, C. Sun, J. H. Snoeijer, and D. Lohse, Maximal Air Bubble Entrainment at Liquid-Drop Impact, Phys. Rev. Lett. 109, 264501 (2012).

[35] H. Gelderblom, H. Lhuissier, A. L. Klein, W. Bouwhuis, D. Lohse, E. Villermaux, and J. H. Snoeijer, Drop deformation by laser-pulse impact (to be published). 


\title{
Supplementary material for Drop shaping by laser-pulse impact
}

\author{
Alexander L. Klein, Wilco Bouwhuis, Claas Willem Visser, Henri Lhuissier, Chao Sun, \\ Jacco H. Snoeijer, Emmanuel Villermaux, Detlef Lohse, and Hanneke Gelderblom
}

Here, we first describe how the energy absorption in the drop is measured. Second, we explain the apparent acceleration of the drop that is visible in Fig. 4 of the main paper "Drop shaping by laser-pulse impact".

\section{MEASUREMENT OF ENERGY ABSORPTION}

We first measure the laser beam profile at the drop location (Fig. 1). To avoid saturation of the beam profiler we attenuate the laser energy by a factor of $10^{6}$ with two wedged windows and a reflective neutral-density filter. To determine the fraction of the light that is refracted into the drop at the air-liquid interface we use ray tracing (valid for $R_{0} \gg \lambda=532 \mathrm{~nm}$ ). Since the dye is highly absorbent $\left(\delta \ll R_{0}\right)$, we neglect any light transmission through the drop. The initial condition of the ray tracing is set by the focal length of the focusing lens and the position of the drop in the laser beam (see Fig. 2 in the main paper). The distribution of rays in space and the energy attributed to each ray is chosen such that the set of rays resembles the measured beam profile. As a result, we estimate that in our experiments $20 \%$ of the laser-pulse energy is absorbed by the drop.

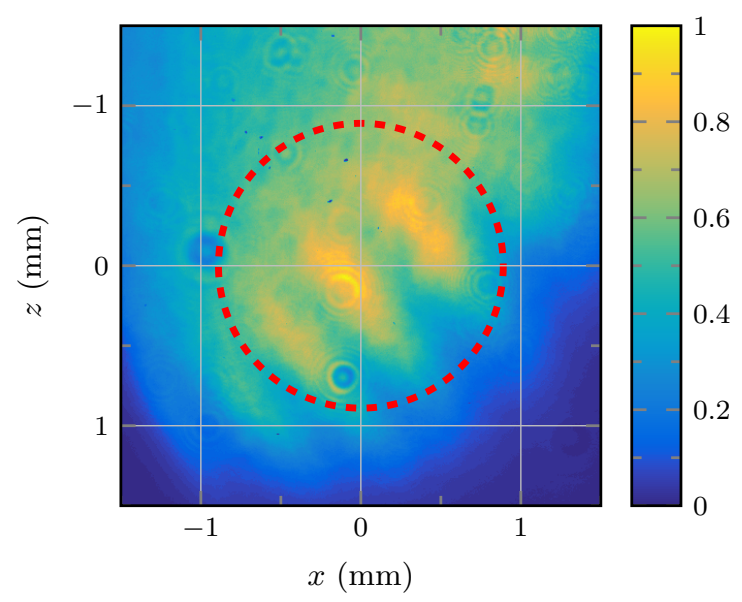

FIG. 1. (Color online) Normalized fluence of the laser beam measured at the drop location by a CCD beam profiler (BC106-VIS by Thorlabs). The profile depicted is the mean result of 10 single measurements. The dotted line indicates the position of the drop during the laser-impact experiments.

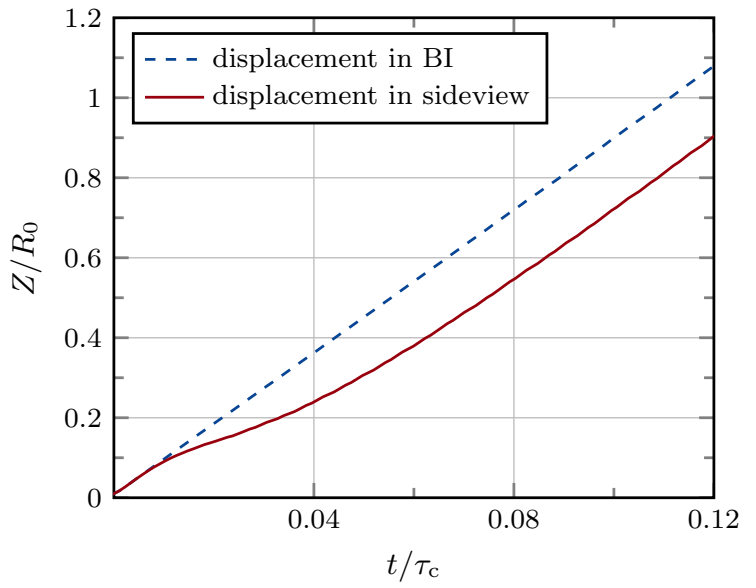

FIG. 2. (Color online) Center-of-mass displacement $Z$ of the drop (along $\vec{e}_{z}$, see Fig. 2 of the main paper) as a function of time in the BI simulation for $E=24 \mathrm{~mJ}$. The blue dashed line represents the actual centre-of-mass displacement, whereas the red solid line is the centre-of-mass displacement based on the two-dimensional projection of the simulated drop shape, which shows an artificial deceleration.

\section{MEASUREMENT OF DROP DISPLACEMENT}

For each recorded image we determine the center-ofmass position assuming rotational symmetry of the twodimensional drop shape. Thereby, we ignore the concavity of the drop shape, which introduces an error in the determination of the centre-of-mass position. This causes an apparent acceleration on the time-scale $\tau_{\mathrm{i}}$ in $Z$ (visible in Fig. 4 of the main paper).

To confirm that this acceleration is an artificial result of our algorithm, we use the results from the BI simulations. In BI the full three-dimensional axi-symmetric shape of the drop is known, and hence the exact centre-ofmass displacement can be determined (dashed, blue line in Fig. 2). In addition, we compute the centre-of-mass displacement based on the two-dimensional projection of the BI data using the same procedure as we apply to the experimental side-view images (solid, red line in Fig. 2). The centre-of-mass determined from the two-dimensional projection shows an artificial deceleration as soon as the drop shape becomes concave. However, Fig. 2 also shows that the final speed of the deforming drop is not affected by the apparent initial deceleration. Therefore, the error introduced by considering the projected drop shape only affects the initial deceleration of the centre-of-mass, not its final speed. 